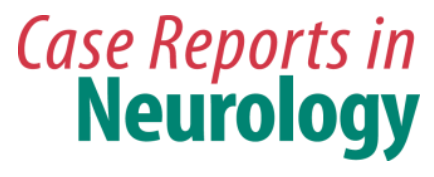

Case Rep Neurol 2016;8:234-242

\title{
Acute Clinical Worsening after Steroid Administration in Cervical Myelitis May Reveal a Subdural Arteriovenous Fistula
}

\author{
Silvia Rain ${ }^{a} \quad J a n U^{\prime} d{ }^{b}{ }^{b}$ Daniel Broere ${ }^{a}$ \\ ${ }^{a}$ Department of Neurology, Westfriesgasthuis Hospital, Hoorn, The Netherlands; \\ ${ }^{b}$ Department of Radiology, Westfriesgasthuis Hospital, Hoorn, The Netherlands
}

\section{Keywords}

Cervical subdural arteriovenous fistula · Cervical myelitis · Quadriplegia · Steroid treatment

\begin{abstract}
Subdural arteriovenous fistula (SDAVF) is a rare condition characterized by clinical manifestations ranging from mild bilateral sensory deficits to quadriplegia. The diagnosis is often delayed due to unspecific neurological symptoms, initially diagnosed as polyneuropathy or myelopathy. The diagnosis can be delayed for as long as 1-15 years. The following report describes a cervical SDAVF case initially misdiagnosed as myelitis transversa and treated with intravenous steroids. A 56-year-old male presented with sensory deficits and mild leg and right arm weakness. Cervical MRI showed a central medullary hyperintense lesion with contrast enhancement. After metabolic, infectious, and malignant causes were excluded, myelitis transversa was presumed and the patient was treated intravenously with methylprednisolone. Shortly after that, he developed quadriplegia. Cervical MRI imaging showed engorged cervical perimedullary vessels, which were not visible on the initial MRI. The diagnosis was revised and a SDAVF identified. Prompt surgical treatment led to a complete recovery. The effect of
\end{abstract}




\section{Case Reports in Neurology}

Case Rep Neurol 2016;8:234-242

DOI: $10.1159 / 000452830$

(c)

(C) 2016 The Author(s). Published by S. Karger AG, Base www.karger.com/crn

Rain et al.: Acute Clinical Worsening after Steroid Administration in Cervical Myelitis May Reveal a Subdural Arteriovenous Fistula

intravenous steroids in SDAVF is controversial. Acute clinical worsening after steroid administration is previously reported in several publications; however, due to the paucity of clinical studies on SDAVF, this effect remains mostly overlooked or unknown. The findings in this patient support the causative relation between SDAVF clinical worsening and steroid administration. We propose that acute clinical worsening under steroids in patients initially diagnosed with myelitis should raise suspicion of an SDAVF.

(C) 2016 The Author(s)

Published by S. Karger AG, Basel

\section{Introduction}

Spinal dural arteriovenous fistula (SDAVF) is a rare neurological disease with an estimated frequency of 5-10 cases per million per year [1]. It is caused by a subdural shunt between a radiculomeningeal artery and a radicular vein, leading to impaired venous drainage from the spinal cord and the arterialization of the vein due to increased venous pressure [2]. It peaks around the 5th-6th decade of life and predominantly affects males. The time to diagnosis is often delayed due to unspecific neurological complaints to as long as 10-15 years after the onset of symptoms [1]. Therefore, $40 \%$ of patients are severely disabled at the time of diagnosis. The most common location of an SDAVF is in the lower thoracic and lumbar region and it is accompanied by complaints in the lower extremities. SDAVF at cervical level (C-SDAVF) is extremely rare, accounting for approximately $2 \%$ of the SDAVF patients and can lead to quadriplegia and respiratory insufficiency.

Early clinical features are nonspecific and include gait claudication, worsened by exercise. By the time of the diagnosis, the triad consisting of (1) motor deficits (leg/arm weakness, muscle cramps), (2) sensory disturbances (paresthesia, numbness, pins and needles sensation, back pain), and (3) cauda syndrome (micturition problems, erectile dysfunction, anal sphincter disturbances) are present in $70 \%$ of the patients [3].

Characteristic MRI features include (1) engorged perimedullary veins, (2) central medullary edema, and (3) swelling of the caudal segments of the spinal cord. In the absence of the findings, diagnosis is difficult to make solely based on clinical symptoms and is usually erroneous [4]. Differential diagnoses may include medullary pathology (myelitis, medullary tumors, disc herniation) or peripheral pathology (sensory polyneuropathy, chronic inflammatory demyelinating polyneuropathy) (Table 1) [5]. Evidence of bladder or bowel dysfunction which share no evident relation with the site of medullary pathology could be very useful in suggesting the diagnosis. The association of cauda symptoms should be considered a "red flag" in SDAVF and can guide the physician toward the correct diagnosis.

Once clinically suspected, SDAVF needs to be radiologically confirmed. The treatment involves either superselective catheterization with endovascular embolization of the fistula or neurosurgical occlusion of the arterialized vein [6]. If successful, the treatment usually stops the progression of symptoms or leads to an improvement of neurological deficits. Therefore, it is of utmost importance that the diagnosis is established as soon as possible in order to avoid permanent handicap. 


\section{Case Reports in Neurology}

Rain et al.: Acute Clinical Worsening after Steroid Administration in Cervical Myelitis May Reveal a Subdural Arteriovenous Fistula

\section{Clinical Case}

A 56-year-old man complained of sensory symptoms (pins and needles sensation) and pain in both legs and the right arm, which progressively worsened in about 4 months. The patient had difficulty performing his daily work as a bus driver and noticed impaired foot sensation and arm weakness during changing gears and pressing the pedals. The symptoms worsened considerably 2 weeks before consulting a neurologist. The patient's past medical history was unremarkable except for hypertension and hypercholesterolemia, both treated with statins and oral antihypertensive drugs. He did not smoke and the family history was unremarkable.

Neurological examination showed generalized weakness in both lower limbs and the right arm (Medical Research Council grade 4) with hyperreflexia and bilateral plantar reflexes. No muscle wasting, atrophy, or fasciculations were observed. Sensory exam showed generalized sensation loss (involving both dorsal columns and the spinothalamic tract) and subjective pins and needles sensations in both feet and the right arm. Additional tests revealed mild urinary retention and decreased sensations in the sacral region.

Given the association of both lower and upper limb involvement and hyperreflexia, a cervical lesion was suspected. The patient underwent diagnostic brain and spinal cord MRI, which showed an elongated intramedullary hyperintensity from the foramen magnum to C4 on T2-weighted images (Fig. 1) with a small contrast enhancement at the junction between the brainstem and the cervical spinal cord. Brain MRI was unremarkable. Additional blood and cerebrospinal fluid laboratory investigations were also unremarkable (Table 2). Transverse myelitis was suspected and the patient was treated with a high intravenous corticosteroid dose [7-10].

Less than $2 \mathrm{~h}$ after the first corticosteroid dose, the symptoms worsened. The patient developed quadriplegia with severe generalized weakness and sensation loss in both legs and both arms, inspiratory muscle weakness, dysphonia, and high urinary retention. An allergic reaction was excluded and the patient was transferred to an intensive care unit for assisted breathing. A cervical MRI was performed showing varicose dilatations, irregular cervical veins, and central medullary edema (Fig. 2) characteristic of a cervical SDAVF [4-6]. The patient was promptly transferred to the neurosurgery department. Superselective angiography confirmed the presence of an SDAVF and the patient successfully underwent open spine surgery with ligation and coagulation of the arterialized communicating vein [6]. Ten days after the surgical treatment, he almost completely regained neurological functions and was further referred to an intensive rehabilitation program in a specialized clinic. By the time of discharge from the neurology ward 2 weeks after surgery, he could walk more than $30 \mathrm{~m}$ with rollator support and only had mild numbness in the feet and some difficulty voiding.

\section{Discussion}

SDAVF may present with a wide range of clinical features such as motor and sensory deficits often associated with a cauda equina syndrome. Neurological deficits are due to perimedullary venous congestion leading to intramedullary edema or ischemia in severe 


\section{Case Reports in Neurology}

Case Rep Neurol 2016;8:234-242

DOI: $10.1159 / 000452830$

(c)

2016 The Author(s). Published by S. Karger AG, Base www.karger.com/crn

Rain et al.: Acute Clinical Worsening after Steroid Administration in Cervical Myelitis May Reveal a Subdural Arteriovenous Fistula

cases or direct compression of the nerve roots at the level of the fistula. Abnormal communication between a radiculomeningeal artery and a radicular vein leads to increased venous pressure in the dural sleeve, impairing normal venous return from the intramedullary capillaries from and below the level of the fistula, causing medullary edema. Furthermore, decreased pressure gradient at capillary level accounts for decreased gas exchange and medullary hypoxia [2].

An acute increase in venous hypertension due to sudden volume or pressure overload may exacerbate neurological deficits by hampering the already decreased venous drainage at the level of the SDAVF causing transitory medullary ischemia. Previous publications report clinical worsening related to an acute increase in intrathoracic and venous pressure such as during singing, Valsalva maneuvers, or abdominal muscle compression during exercise [11].

In addition to the current paper, several publications report rapid worsening of symptoms in patients receiving corticosteroids intravenously for other presumed diagnoses (most commonly myelitis) [12-16]. Although the exact mechanism which leads to worsening of spinal cord dysfunction is not yet understood, it is possible that both corticosteroid and intravenous saline in which these are administered could play a role. In addition to antiinflammatory effects, corticosteroids can also play a role in regulating water and mineral metabolism through their mineralocorticoid effects, causing fluid retention. This can compromise the already fragile venous return into the dural sac, leading to medullary hypoxia and further worsening the SDAVF symptoms [17]. Furthermore, steroids administered via an intravenous line placed in the upper extremity eventually drain via the cephalic veins into the brachiocephalic veins. The azygos and semiazygos veins, which collect venous blood from the spinal cord, are also tributaries of the brachiocephalic veins. Therefore, a rapid rise in venous return from the upper extremities into the brachiocephalic vein could also directly diminish the venous return from the medullary level and exacerbate symptoms.

This theory is supported by the MRI findings before and after corticoid treatment in our patient. The cervical MRI before intravenous treatment shows no evident perimedullary venous abnormalities, whereas after treatment, engorged veins can clearly be identified in the subdural space, as evidence of acute venous hypertension in the subdural sac (Fig. 3).

\section{Conclusion}

We would like to conclude this study by stressing out the importance of early diagnosis and treatment in patients with SDAVF. However, due to nonspecific clinical and radiologic findings, this may be challenging. Therefore, the presence of "red flags" can be extremely useful in guiding the physician toward the correct diagnosis. Acute neurological worsening after intravenous steroid administration for any presumed diagnosis should be considered such a "red flag" and raise the suspicion of an SDAVF.

\section{Statement of Ethics}

The current manuscript is in accordance to the Medical Ethics. 


\section{Case Reports in Neurology}

Case Rep Neurol 2016;8:234-242

DOI: $10.1159 / 000452830$

(C) 2016 The Author(s). Published by S. Karger AG, Basel www.karger.com/crn

Rain et al.: Acute Clinical Worsening after Steroid Administration in Cervical Myelitis May Reveal a Subdural Arteriovenous Fistula

\section{Disclosure Statement}

\section{There is no conflict of interest.}

\section{References}

1 Jellema K, Canta LR, Tijssen CC, van Rooij WJ, Koudstaal PJ, van Gijn J: Spinal dural arteriovenous fistulas: clinical features in 80 patients. J Neurol Neurosurg Psychiatr 2003;74:1438-1440.

-2 Krings T, Geibprasert S: Spinal dural arteriovenous fistulas. AJNR Am J Neuroradiol 2009;30:639-648.

-3 Muralidharan R, Saladino A, Lanzino G, Atkinson JL, Rabinstein AA: The clinical and radiological presentation of spinal dural arteriovenous fistula. Spine 2011;36:E1641-E1647.

-4 Jeng Y, Chen DYT, Hsu HL, Huang YL, Chen CJ, Tseng YC: Spinal dural arteriovenous fistula: imaging features and its mimics. Korean J Radiol 2015;16:1119-1131.

5 Jellema K, Tijssen CC, van Gijn J: Spinal dural arteriovenous fistulas: a congestive myelopathy that initially mimics a peripheral nerve disorder. Brain 2006;129:3150-3164.

6 Kim DJ, Willinsky R, Geibprasert S, Krings T, Wallace C, Gentili F, Terbrugge K: Angiographic characteristics and treatment of cervical spinal dural arteriovenous shunts. AJNR Am J Neuroradiol 2010;31:1512-1515.

7 Jacob A, McKeon A, Nakashima I, Sato DK, Elsone L, Fujihara K, de Seze J: Current concept of neuromyelitis optica (NMO) and NMO spectrum disorders. J Neurol Neurosurg Psychiatr 2013;84:922930. Chang KH, Lyu RK, Chen CM, Wu YR, Chang HS, Huang CC, Kuo HC, Chu CC, Hsu WC, Ro LS: Distinct features between longitudinally extensive transverse myelitis presenting with and without antiaquaporin 4 antibodies. Mult Scler 2013;19:299-307. Sellner J, Boggild M, Clanet M, Hintzen RQ, Illes Z, Montalban X, Du Pasquier RA, Polman CH, Sorensen PS, Hemmer B: EFNS guidelines on diagnosis and management of neuromyelitis optica. Eur J Neurol 2010;17:1019-1032. Kitley JL, Leite MI, George JS, Palace JA: The differential diagnosis of longitudinally extensive transverse myelitis. Mult Scler 2012;18:271-285.

11 Bernstein RA, Gress DR: Dural arteriovenous fistulas and the neurology of venous hypertension. Semin Cerebrovasc Dis Stroke 2004;4:168-175.

12 Strowd RE, Geer C, Powers A, Abou-Zeid N: A unique presentation of a spinal dural arteriovenous fistula exacerbated by steroids. J Clin Neurosci 2012;19:466-468.

13 Cabrera M, Paradas C, Márquez C, González A: Acute paraparesis following intravenous steroid therapy in a case of dural spinal arteriovenous fistula. J Neurol 2008;255:1432-1433.

14 Nasr DM, Brinjikji W, Rabinstein AA, Lanzino G: Clinical outcomes following corticosteroid administration in patients with delayed diagnosis of spinal arteriovenous fistulas. J Neurointerv Surg 2016;10:113.

15 O'Keeffe DT, Mikhail MA, Lanzino G, Kallmes DF, Weinshenker BG: Corticosteroid-induced paraplegia a diagnostic clue for spinal dural arterial venous fistula. JAMA Neurol 2015;72:833-834.

16 McKeon A, Lindell EP, Atkinson JLD, Weinshenker BG, Piepgras DG, Pittock SJ: Pearls and oy-sters: clues for spinal dural arteriovenous fistulae. Neurology 2011;76:e10-e12.

17 Winter DC, Schneider MF, O'Sullivan GC, Harvey BJ, Geibel JP: Rapid effects of aldosterone on sodiumhydrogen exchange in isolated colonic crypts. J Membr Biol 1999;170:17-26. 


\section{Case Reports in Neurology}
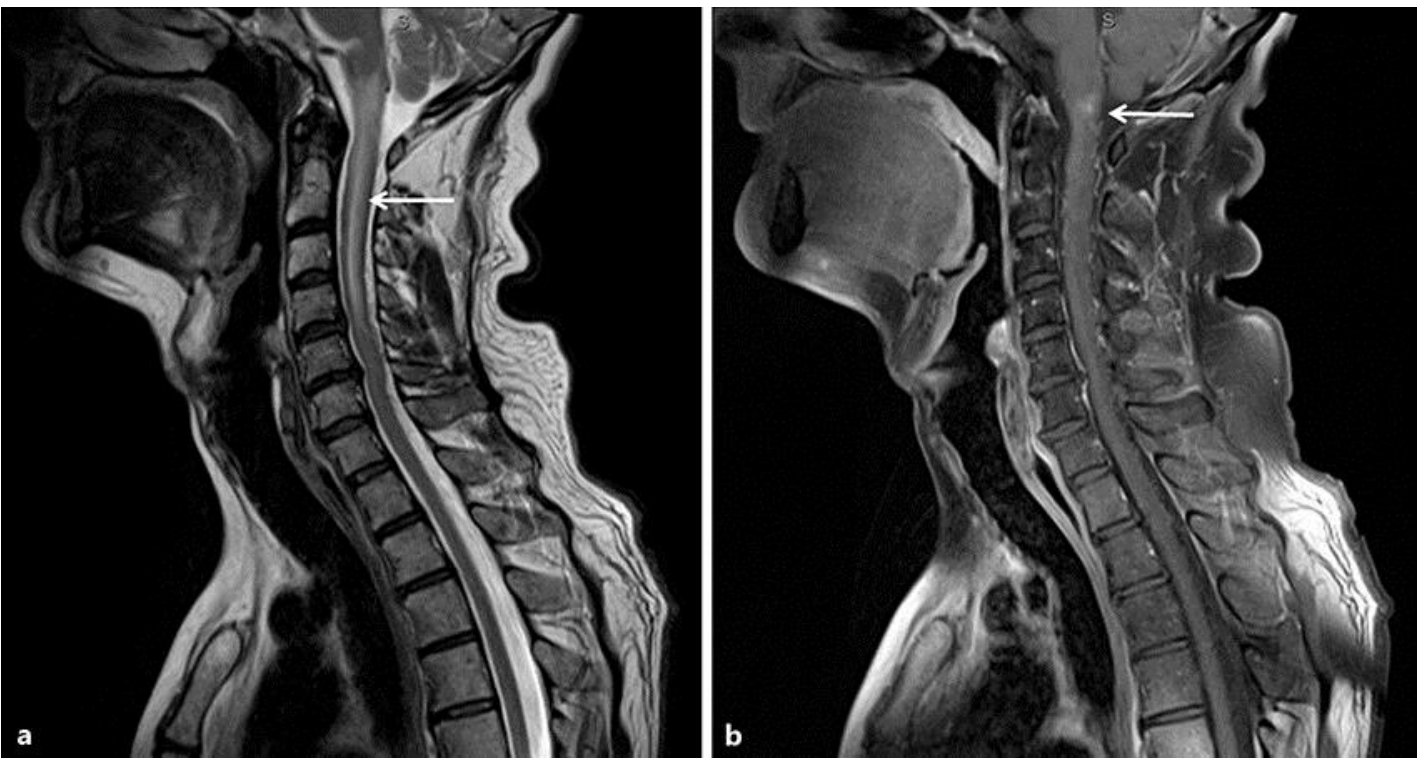

Fig. 1. Admission spinal MRI. a Sagittal T2-weighted image showing central medullary hyperintensity (white arrow) elongated from the foramen magnum to the fourth cervical vertebral body. b Sagittal T1weighted image with gadolinium enhancement showing a small dotted region of contrast enhancement above the first cervical vertebral body (white arrow). 


\section{Case Reports in Neurology}

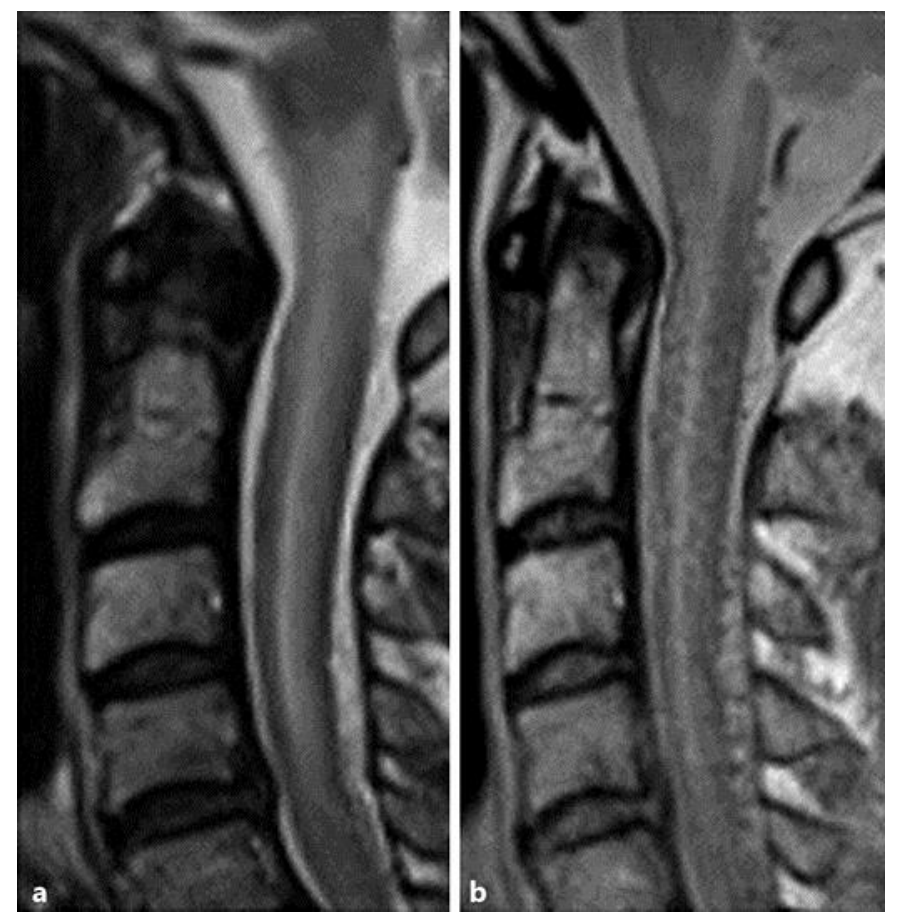

\begin{tabular}{l|l} 
DOI: $10.1159 / 000452830$ & (c 2016 The Author(s). Published by S. Karger AG, Basel
\end{tabular} www.karger.com/crn

Rain et al:: Acute Clinical Worsening after Steroid Administration in Cervical Myelitis May Reveal a Subdural Arteriovenous Fistula

Fig. 3. Medullary changes before and after steroid administration. a Sagittal T2-weighted image of the cervical region before intravenous steroid administration. b Sagittal T2-weighted image of the cervical region after intravenous steroid administration. 
Table 1. Differential diagnosis of SDAVF

\section{Myelitis}

Transverse myelitis/neuromyelitis optica spectrum disorder

Multiple Sclerosis

Inflammatory diseases/vasculitis: Sjögren's syndrome, sarcoidosis, systemic lupus erythematosus

Infectious cause: varicella-zoster virus, cytomegalovirus, Epstein-Barr virus, syphilis, human

immunodeficiency virus, dengue, hepatitis A virus, mycoplasma pneumoniae, mycobacterium tuberculosis, parasitary

\section{Myelopathy}

Malignancy: intramedullary tumors (ependymoma, astrocytoma), paraneoplastic syndromes

Vascular disease: spinal cord infarcts, spinal dural arteriovenous fistula

Metabolic disease: vitamin B12 deficiency, copper deficiency

3. Polyneuropathy

Malignancy: paraneoplastic syndrome

Infectious cause: HIV, Borrelia burgdorferi

Metabolic disease: diabetes mellitus, alcohol abuse, liver failure, vitamin $\mathrm{B}_{12} /$ folic acid deficiency, hypothyroid disease

Table 2. Additional laboratory investigations

\section{Blood tests}

Standard lab: normal

ANA, ANCA, ACE, lupus anticoagulant, onconeural antibodies:

negative

Vitamin $B_{12}$, copper: normal

Aquaporin 4 antibodies: negative

Borrelia burgdorferi antibodies: negative

Syphilis infection: negative

HIV infection: negative

\section{Cerebrospinal fluid tests}

Erythrocytes 41, leukocytes 6, glucose 3.6, total protein 434, IgG 20, albumin 309, IgG index: normal

Oligoclonal bands: negative

Borrelia burgdorferi antibodies: negative, neurotropic viruses

PCR: negative

Pathology exam: benign cells 